\title{
On the neurology of morals
}

\author{
Raymond J. Dolan
}

\begin{abstract}
Patients with medial prefrontal lesions often display irresponsible behavior, despite being intellectually unimpaired. But similar lesions occurring in early childhood can also prevent the acquisition of factual knowledge about accepted standards of moral behavior.
\end{abstract}

The story of Phineas Gage is a landmark case in neurology ${ }^{1}$. After a penetrating prefrontal injury (Fig. 1), which mainly damaged the orbitofrontal cortex (OFC), the personality of this formerly conscientious railway worker deteriorated profoundly, with devastating implications for his social adjustment. Though superficially normal, Gage was unable to fit into his previous social and occupational roles. He became unreliable in his work and showed a callous disregard for his loved ones. One of the striking features of OFC damage first highlighted in this case is that gross behavioral changes occurred despite otherwise preserved intellectual function. Later reports of patients with similar damage showed that this preserved intellectual function extends to performance on psychological tasks that tap prototypical frontal lobe functions such as working memory and planning.

In the current issue of Nature Neuroscience, Anderson and colleagues ${ }^{2}$ describe two patients in whom the ventral prefrontal sector of prefrontal cortex (PFC) was damaged early in life (Fig. 2). Both patients showed lifelong behavioral problems resistant to corrective influences. Most importantly, they also specifically lacked knowledge regarding societal and moral conventions that are fundamental factors in behavioral regulation. The detailed descriptions of these two cases are challenging in two respects. First, they extend the suggested range of functions subsumed by PFC. Second, they have profound implications for what constitutes the legitimate domain of cognitive neuroscience.

One of the most important issues in this field is the identification of factors that influence behavioral choice. PFC is widely believed to be critically involved in this process. Within this region, there are at least three different functional zones: dorsolateral, dorsomedial and orbitofrontal cortices.

Raymond Dolan is at the Wellcome Department of Cognitive Neurology, Institute of Neurology, 12 Queen Square, London WC1N 3BG, United Kingdom.

Email: r.dolan@fil.ion.ucl.ac.uk
The relative contribution of each of these zones to goal-directed behavior is still unresolved. This issue of specialization of function within PFC has been the focus of much recent research. For example, many distinct psychological functions can be attributed to dorsolateral and dorsomedial sectors, including components of attention, memory, planning and monitoring. Coordination of these and other (as yet unspecified) functions within PFC presumably provides the basis for behavior that is flexible and adaptable in relation to present and future environmental contingencies.

Defining the contributions of OFC to behavioral regulation has proved most elusive. This region, located on the ventral aspect of PFC above the eye sockets, bears the brunt of the damage in the two cases reported by Anderson and colleagues ${ }^{2}$. This group, led by Antonio Damasio, has been at the forefront of the endeavor to characterize the functions of human OFC. Damage to this zone in adulthood has profound effects on emotional expression, personality and behavior. Anderson and colleagues asked whether damage to OFC acquired in early life has similar behavioral and psychological consequences.

Given that damage to this region affects personality and behavior, there are formidable obstacles to its study in the laboratory. To overcome this difficulty, this group has devised specific laboratory tasks that mimic real-life situations, such as their well-known gambling task. This task involves selecting cards from one of four decks that are associated with varying schedules of reward and punishment, which subjects do not know in advance. When asked to choose randomly and repeatedly from decks, neurologically intact individuals, and indeed patients with damage to dorsolateral PFC, maximize their gains by showing an increasing bias toward advantageous decks. By contrast, patients with adult-acquired OFC lesions show no such bias and continue to select disadvantageously in a manner driven by immediate rather than long-term rewards ${ }^{3-5}$. Anderson and colleagues ${ }^{2}$ show that patients with early-acquired OFC damage behave similarly. Intriguingly, adult OFC patients can verbally describe the most appropriate strategy for optimal task performance, but they cannot effectively use this knowledge in performing the task.

Patients with early-acquired OFC damage $^{2}$ are remarkably similar to those with adult-acquired damage in that they display profound and pervasive difficulties in social behavior despite intact intellectual abilities. These adjustment problems were obvious from an early age. However, their problems include stealing and violence to persons, which the authors report is not characteristic of

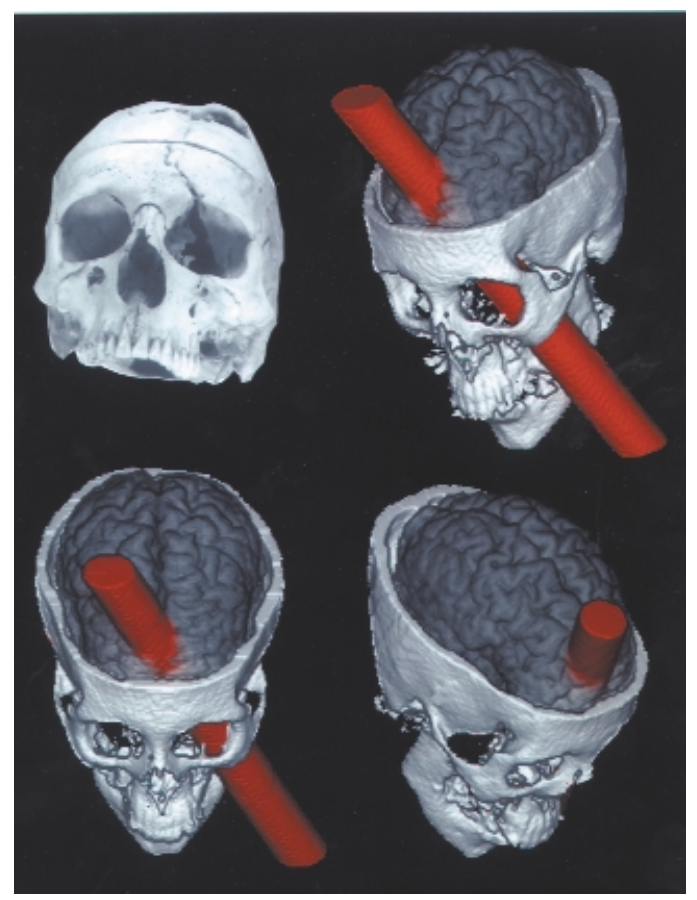

Fig. I. Reconstruction of the lesion incurred by Phineas Gage, in which an iron bar was driven through his prefrontal cortex as a result of a blasting accident. Courtesy of Hannah Damasio. 


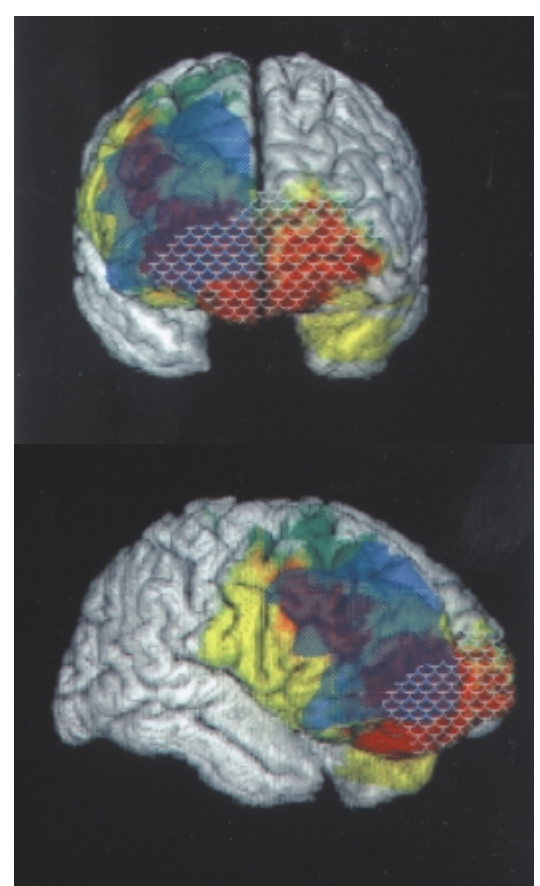

Fig. 2. MAP-3 overlap showing regions of frontal lobe damaged by lesions in early childhood (cross-hatched) or adulthood (colored). Courtesy of Hannah Damasio.

adult-onset patients. In this respect, the early-onset patients resemble psychopathic individuals, who are characterized by high levels of aggression and antisocial behavior performed without guilt or empathy for their victims. The most crucial difference from patients injured in adulthood was found on tasks measuring moral reasoning and verbal responses to social situations. These standardized tasks present various real-life scenarios, followed by questions about the conflicts or dilemmas posed. In contrast to adultonset patients, the performance of both early-damaged patients on such tasks was markedly defective. Indeed, their moral reasoning was at a level appropriate for age ten, being characterized by an excessively egocentric perspective. In the authors' view, their deficits reflect a failure to acquire social and moral knowledge. The remarkable implication of these findings is that the integrity of the OFC is necessary for acquiring very specific forms of knowledge necessary for regulating interpersonal and social behavior.

These findings raise a number of caveats and questions. First, there is the limited sample size. However, such patients are exceedingly rare, and the current report is the most comprehensive to date on long-term consequences of early damage to OFC and adjacent cortex. Second, although early OFC lesions result in a behavioral deficit similar to that found after adult damage, it would nevertheless be of interest to know the outcome of early lesions to other prefrontal sectors. This is clearly an issue for future research. In addition, the lesions in the present patients involve different segments of OFC and related cortices. This variability might be interpreted to indicate a lack of specificity. An equally likely possibility is that lesions in different locations, through effects on white matter connections, compromise a common functional system.

To put these new findings in perspective, it is useful to consider existing theories of prefrontal cortex function. Luria proposed that PFC acts to integrate all stimuli reaching the organism and attach their informative or regulatory significance $^{6}$. Nauta believed patients with orbitofrontal damage suffer from ignorance of somatic bodily symptoms that are critical cues to the meaning or value of situations or thoughts ${ }^{7}$. However, Damasio $^{8}$ has articulated the most comprehensive account of OFC function. His 'somatic-marker hypothesis' proposes that behavioral deficits following OFC damage reflect an inability to involve emotional processing in the response to complex task situations. Emotional influences are held to act as a biasing signal, such that when an individual contemplates options for action, the OFC engages knowledge related to feelings engendered by similar situations in the past. This information is used to select the action that is likely to be optimally advantageous. This type of biasing signal has the most influence when the future is uncertain. In patients with OFC lesions, this mechanism is compromised, accounting for undue reliance on immediate, as opposed to future, advantage and failure to adjust behavior based on past experience. This theory's elegance is that it provides the crucial link between emotion and behavior that can account for behavioral deficits found in OFC patients.

The somatic-marker hypothesis, derived largely from human lesion studies, has been strongly endorsed by other approaches. Thus, an emerging theme, particularly in electrophysiological investigations, is that the OFC is involved in the prospective representation of the reward value of behavior options. For instance, in monkeys, the activity of neurons in the OFC increases in relationship to reward-predicting signals ${ }^{9}$, with different responses depending on whether cues predict a positive or negative outcome ${ }^{10}$. After lesions of OFC, rats are unable to access the incentive value of an associated reinforcement in response to cues ${ }^{11}$. Finally, human functional neuroimaging data indicate that OFC is involved in appraising the reward value of ongoing behavior, particularly under unpredictable circumstances $^{12}$.

The new observation in patients with early-acquired OFC lesions is that their behavioral deficits are accompanied by an absence of factual knowledge regarding expected social and moral norms. Why specific forms of knowledge acquisition should be compromised is not an easy question to answer. Anderson and colleagues $^{2}$ suggest that this impairment may emerge from destruction of a necessary cortical region that influences learning through reward and punishment. The implication is that acquisition of social and moral knowledge may depend critically on being able to experience feelings evoked by reward and punishment. The specificity of deficits for the social-moral domain could conceivably depend on a deficit in the modulation of knowledge acquisition by feelings evoked during interpersonal encounters.

The findings of Anderson and colleagues ${ }^{2}$, coupled with the neurophysiological and neuroimaging data discussed above, converge on the idea that OFC codes the likely significance of future behavioral options. This function must rely to a large extent on an evaluation of whether similar past behavioral options were associated with reward or punishment. Representing the probable outcomes of options for action, by reference to recent and past experience, may consequently provide incentive values crucial to behavioral choice. The difficult problem raised by the new findings is accounting for how these processes might extend to the specific domain of knowledge acquisition that provides a basis for moral and social behavior. As already suggested, the crucial element may involve a deficit in representing the value of behavioral options that involve other people. The importance of these issues is that they suggest that knowledge acquisition relevant to moral and social behavior has a neurobiological substrate dissociable from that of other forms of knowledge acquisition. If this proves to be correct, we will need to reappraise the societal challenges posed by psychopathy and related disorders, which currently (and conveniently) linger in a hinterland between psychiatry and criminal services. 
1. Harlow, J. M. Boston Med. Surg. J. 39, 389-393 (1848).

2. Anderson, S. W., Bechara, A., Damasio, H., Tranel, D. \& Damasio, A. R. Nat. Neurosci. 2, 1032-1037 (1999).

3. Damasio, A. R. \& Van Hoesen, G. W. in Neuropsychology of Human Emotion (eds. Heilman, K. M. \& Satz, P.) 85-110 (Guilford, New York, 1983).
4. Damasio, A. R., Tranel, D. \& Damasio, H. Behav. Brain Res. 41, 81-94 (1990).

5. Bechara, A., Damasio, A. R., Damasio, H. \& Anderson, S. W. Cognition 50, 7-15 (1994).

6. Luria, A. R. The Higher Cortical Functions In Man (Basic Books, New York, 1980).

7. Nauta, W. J. H. J. Psychiatr. Res. 8, 167-187 (1971).

8. Damasio, A. Descartes' Error (Grosset/Putnam, New York, 1994).

\section{UNC-13 and neurotransmitter release}

\author{
Hiroshi Tokumaru and George J. Augustine
}

\section{Genetic studies in mice, worms and flies indicate that the synaptic protein UNC-13 plays a central role in the regulation of vesicle fusion.}

Evoked synaptic transmission involves the fusion of synaptic vesicles with the presynaptic membrane in response to transient increases in presynaptic calcium concentration. This process involves a complex molecular apparatus, several components of which have now been identified and assigned tentative functions. The fusion reaction itself apparently requires the SNARE proteins - syntaxin, SNAP-25 and synaptobrevin $^{1-3}$ — whereas synaptotagmin is widely thought to be a calcium receptor protein that triggers fusion ${ }^{1,2}$. Before synaptic vesicles fuse, however, they must first dock at the plasma membrane and undergo priming reactions that make both membranes competent for fusion. Precisely what happens during these docking and priming steps is not clear. Now three excellent papers ${ }^{4-6}$, two of them in this issue, implicate another protein, UNC-13, in the events that lead to vesicle fusion.

UNC-13 was first identified in the nematode Caenorhabditis elegans by the classical genetic screening efforts of Brenner ${ }^{7}$. He found that mutations in a number of genes, including $u n c-13$, caused the worms to exhibit severely uncoordinated movements, suggesting that UNC-13 has a neural function. UNC-13 is a large protein (approximately $200 \mathrm{kDa}$ molecular weight) that is neuron-specific and phylogenetically conserved ${ }^{4-6}$. It contains a number of motifs found in other pro-

The authors are at the Department of Neurobiology, Duke Medical Center, Durham, North Carolina 27710 and Marine Biological Laboratory, Woods Hole, Massachusetts 02543, USA.

e-mail: georgea@neuro.duke.edu teins ${ }^{8}$, providing clues to its possible function. For example, the presence of $\mathrm{C} 2$ domains suggest that UNC-13 may bind calcium, although this has yet to be tested directly. UNC-13 also has a C1 domain, which (like that of protein kinase $\mathrm{C}$ ) binds to diacylglycerol (DAG), an endogenous second messenger, as well as to phorbol esters, which mimic the effect of DAG. Both DAG and phorbol esters can stimulate transmitter release, and this effect seems to involve UNC-13; treatment with DAG or phorbol esters causes UNC-13 to be translocated to the plasma membrane $^{9}$, and overexpression ${ }^{9}$ or blockade ${ }^{10}$ of UNC-13 increases or decreases the sensitivity of neurotransmitter release to phorbol esters, respectively. Although these experiments showed that UNC-13 is able to regulate neurotransmitter release, they could not determine whether UNC-13 indeed mediates transmitter release in vivo.

The three new papers have tackled this question by examining the effect of knocking out the unc-13 genes in mice $^{4}$, C. elegans 5 and Drosophila 6 . This produced lethal phenotypes in Drosophila and mice and severely uncoordinated movement in C. elegans. High-resolution electrophysiological recordings were used to analyze synaptic transmission in mutant neurons, at stages before the lethal phenotypes killed the animals.
9. Tremblay, L. \& Schultz, W. Nature 398, 704-708 (1999).

10. Schoenbaum, G., Chiba, A. A. \& Gallagher, M. Nat. Neurosci. 1, 155-159 (1998).

11. Gallagher, M., McMahan, R. W. \& Schoenbaum, G. J. Neurosci. 19, 6610-6614 (1999).

12. Elliott, R., Frith, C. D. \& Dolan, R. J. Neuropsychologia 247, 1395-1404 (1997).

In particular, measurements of neurotransmitter release at the level of individual quanta ${ }^{11}$ were helpful for deducing the mechanisms underlying changes in transmitter release found in mutant synapses.

Despite the range of organisms analyzed, the main findings of the three studies were remarkably coherent. In all cases, loss of UNC-13 greatly reduced the amount of neurotransmitter release evoked by presynaptic action potentials. In C. elegans, strong alleles reduced synaptic transmission to $0-6 \%$ of controls. For the Drosophila and mouse mutations, evoked glutamate release was reduced to below $1 \%$ or $10 \%$, respectively, of wildtype levels. These changes in release were due to a reduced number of quanta released from the presynaptic terminals, rather than a change in the size of individual quanta. Thus, UNC-13 is important for the release of transmitters rather than their packaging into synaptic vesicles.

The spontaneous release of transmitter that occurs in the absence of presynaptic stimulation was also dramatically reduced by the loss of UNC-13. In C. elegans mutants, the frequency of these spontaneous events was reduced to less than $1 \%$ of wild-type levels. Likewise, in Drosophila mutants these events occurred at approximately $25 \%$ of control frequencies. In mutant mice, the frequency of spontaneous glutamatergic events was approximately $5 \%$ of control levels. This indicates that UNC-13 is involved not

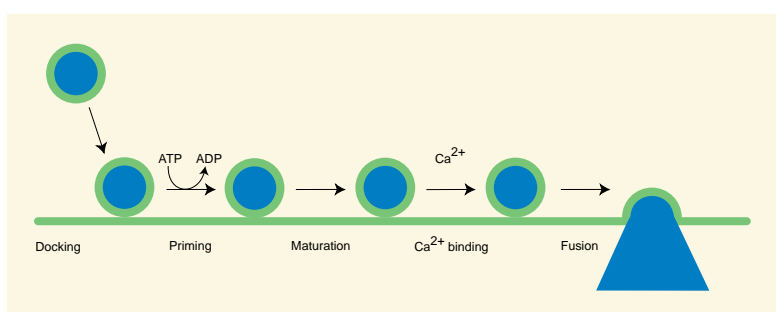

Fig. I. Reactions that lead to synaptic vesicle fusion. Vesicles dock at the plasma membrane and undergo ATP-dependent priming reactions to become fusion competent. Additional 'maturation' reactions may also be required for docked vesicles to prepare for fusion. After membrane depolarization causes calcium entry into the presynaptic terminal, one or more proteins rapidly bind calcium to trigger the final membrane fusion reaction and discharge of neurotransmitter. 